\title{
Review: psychoeducational programmes reduce long term mortality and recurrence of myocardial infarction in cardiac patients
}

Dusseldorp E, van Elderen T, Maes S, et al. A meta-analysis of psychoeducational programs for coronary heart disease patients. Health Psychol 1999 Sep;18:506-19.

QUESTION: Can psychoeducational programmes (stress management and health education) reduce cardiac morbidity, mortality, and risk factors in patients with coronary artery disease $(\mathrm{CAD})$ ?

\section{Data sources}

Studies were identified by searching Medline and PsycLIT (1974-98) and by scanning bibliographies of relevant articles.

\section{Study selection}

Published studies were selected if they involved patients who had had a cardiac event (myocardial infarction [MI], coronary artery bypass grafting, percutaneous transluminal coronary angioplasty, or combination) within 6 months before treatment and if the study design was a randomised controlled trial (RCT) or quasi-randomised trial. Studies reporting effects on emotional distress were included only if effects on risk factors, related behaviours, morbidity, or cardiac mortality were also reported. Quasirandomised trials were included only if samples were stratified or matched pairwise or if patients from the same hospital were allocated by using time periods.

\section{Data extraction}

2 reviewers independently abstracted data on the type and duration of intervention, patient characteristics, setting, year of publication, study methods, and effect sizes for cardiac events and health outcomes. When reviewers disagreed, a 3rd reviewer made the final decision.

\section{Main results}

37 studies (28 RCTs) published from 1974-97 met the inclusion criteria. The duration of the interventions ranged from $<6$ weeks to $>6$ months (mean duration 28 wks). Interventions were health education, stress management, or both, and these were sometimes combined with exercise training. Patients in the control group received standard care (30 studies), exercise training (6 studies), or health education ( 1 study). The combined results showed that psychoeducational programmes led to (1) a $34 \%$ relative reduction in cardiac mortality over the long term (>2 y) (6 studies), (2) a 29\% relative reduction in MI recurrence at 2-10 years follow up (7 studies), and (3) improved risk factor profiles for systolic blood pressure (8 studies), total serum cholesterol (7 studies), weight (8 studies), and smoking over the short and medium terms (follow up 6 wks to 2 y) (21 studies). Psychoeducational programmes did not have an effect on the following outcomes: coronary artery bypass grafting (9 studies), anxiety (10 studies), or depression (13 studies). Success on proximal targets (eg, risk factors) was found to be an important moderator of success on distal outcomes (eg, cardiac death and recurrence of angina and MI).

\section{Conclusion}

In patients with coronary artery disease, psychoeducational programmes help decrease long term cardiac mortality, myocardial infarction recurrence, and major cardiac risk factors (systolic blood pressure, total serum cholesterol, weight, and smoking behaviour).

\section{COMMENTARY}

This meta-analysis by Dusseldorp et al reinforces previous findings that psychoeducational interventions reduce major cardiac risk factors, and that this reduction contributes to decreases in cardiac death, angina, and MI. A unique finding was that patients who had received a psychoeducational programme were 3 times more likely to quit smoking than those who had not. Two other reviews differed: Mullen et al reported no significant programme effect on smoking behaviour, ${ }^{1}$ and Ebrahim and Smith observed a $4.2 \%$ reduction in smoking prevalence rates. ${ }^{2}$ Many studies report inconsistent results for smoking cessation; success has been associated with a desire to stop, a life threatening cardiac event, advice to stop, and support. ${ }^{3}$

Dusseldorp et al also reported no significant programme effect on anxiety or depression; this finding contrasted with Linden et al's study review which showed reductions in psychological stress after psychosocial interventions. ${ }^{4}$ Specific programmes that target the most highly stressed patients with CAD have been beneficial. ${ }^{5}$

Dusseldorp et al's results have important clinical implications for patients, nurses, and other health professionals who work in inpatient and community settings. The findings reinforce the view that participation in cardiac rehabilitation programmes, which use stress management and health education interventions, results in clinical benefits. Nurses have a substantive role in the planning, implementation, and evaluation of educational programmes. This review provides a stimulus for them to collaborate with their patients in choosing evidence-based behavioural strategies for secondary prevention after a cardiac event.

The effectiveness of psychoeducational programmes upon anxiety and depression needs further clarification. More research targeting specific groups (different sexes, ages, and cultures) needs to be done.

The findings of the review by Dusseldorp et al provide encouragement to nurses to continue their role in psychoeducational programmes as part of cardiac rehabilitation.

Gerry Benson, RN, MSc Assistant Professor, School of Nursing McMaster University

Hamilton, Ontario, Canada

1 Mullen PD, Mains DA, Velez R. A meta-analysis of controlled trials of cardiac patient education. Patient Educ Coun 1992;19:143-62.

2 Ebrahim S, Smith GD. Systematic review of randomised controlled trials of multiple risk factor interventions for preventing coronary heart disease. BMJ 1997;314:166674

3 Clark JM, Haverty S, Kendall S. Helping people to stop smoking: a study of the nurse's role. J Adv Nurs 1990;15:35763.

4 Linden W, Stossel C, Maurice J. Psychosocial interventions for patients with coronary artery disease: a meta-analysis Arch Intern Med 1996;156:745-52.

5 Milani RV, Lavie CJ. Prevalence and effects of cardiac rehabilitation on depression in the elderly with coronary heart disease. Am J Cardiol 1998;81:1233-6. 